\title{
A new lichenized fungus, Lecanora baekdudaeganensis, from South Korea, with a taxonomic key for Korean Lecanora species
}

\author{
Beeyoung Gun Lee', Jae-Seoun Hur² \\ I Baekdudaegan National Arboretum, Bonghwa, 36209, South Korea 2 Korean Lichen Research Institute, \\ Sunchon National University, Suncheon 57922, South Korea \\ Corresponding author: Jae-Seoun Hur (jshur1@scnu.ac.kr)
}

Academic editor: T. Lumbsch | Received 28 February 2020 | Accepted 15 June 2020 | Published 24 July 2020

Citation: Lee BG, Hur J-S (2020) A new lichenized fungus, Lecanora baekdudaeganensis, from South Korea, with a taxonomic key for Korean Lecanora species. MycoKeys 70: 39-58. https://doi.org/10.3897/mycokeys.70.51569

\begin{abstract}
Lecanora baekdudaeganensis Lee \& Hur is described as a new lichenized fungus from Baekdudaegan Mountains, South Korea. The new species is classified into the Lecanora subfusca group - allophana type and distinguishable from Lecanora imshaugii Brodo by a darker thallus, brownish disc, K-insoluble granules on the surface of the epihymenium, shorter hypothecium, and the presence of oil droplets in the apothecial section. Molecular analyses employing internal transcribed spacer (ITS) and mitochondrial small subunit (mtSSU) sequences strongly support Lecanora baekdudaeganensis as a distinct species in the genus Lecanora. A surrogate key is provided to assist in the identification of all 52 taxa in the genus Lecanora of Korea.
\end{abstract}

\section{Keywords}

biodiversity, Lecanoraceae, phorophyte, phylogeny, taxonomy

\section{Introduction}

The Baekdudaegan Mountains are the main mountain range stretching across the entire Korean Peninsula. The mountains stretch 1,400 km in length from North Korea to South Korea and encompass protected areas of approximately $2,750 \mathrm{~km}^{2}$ (Korea Forest Service 2019). The Baekdudaegan Mountains, as the main mountain system for whole mountainous areas comprising 70 percent of Korea, are almost totally covered with forest and support a productive ecosystem for specialists as well as generalists, 
represented by 27 percent endemic vascular plants (Korea Forest Service 2019) and 20 percent endemic lichens/lichenicolous fungi.

Although the genus Lecanora is one of the largest genera in lichens, just three new species in Lecanora were formerly discovered out of all 164 lichenized or lichenicolous fungi which were reported as new species from Korea. Specifically, all three species, $L$. hafelliana L. Lü, Y. Joshi \& Hur, L. loekoesii L. Lü, Y. Joshi \& Hur, and L. psendosambuci S.Y. Kondr., Lőkös \& Hur were detected from the bark of Quercus or other deciduous trees in the Baekdudaegan mountains or other mountainous areas in North Korea and South Korea (Lü et al. 2011; Kondratyuk et al. 2016) (Fig. 1).

This study describes a new lichenized fungus in the genus Lecanora. During three field trips to Mt. Munsu, Bonghwa in 2019 (Fig. 1), four specimens were collected but identified just to genus without matching any previously known species. We describe them below as a new corticolous lichen species, Lecanora baekdudaeganensis, and this discovery contributes to the taxonomy with overall 52 taxa in the genus Lecanora from North Korea and South Korea. All specimens are deposited in the herbarium of the Baekdudaegan National Arboretum (BDNA), South Korea.

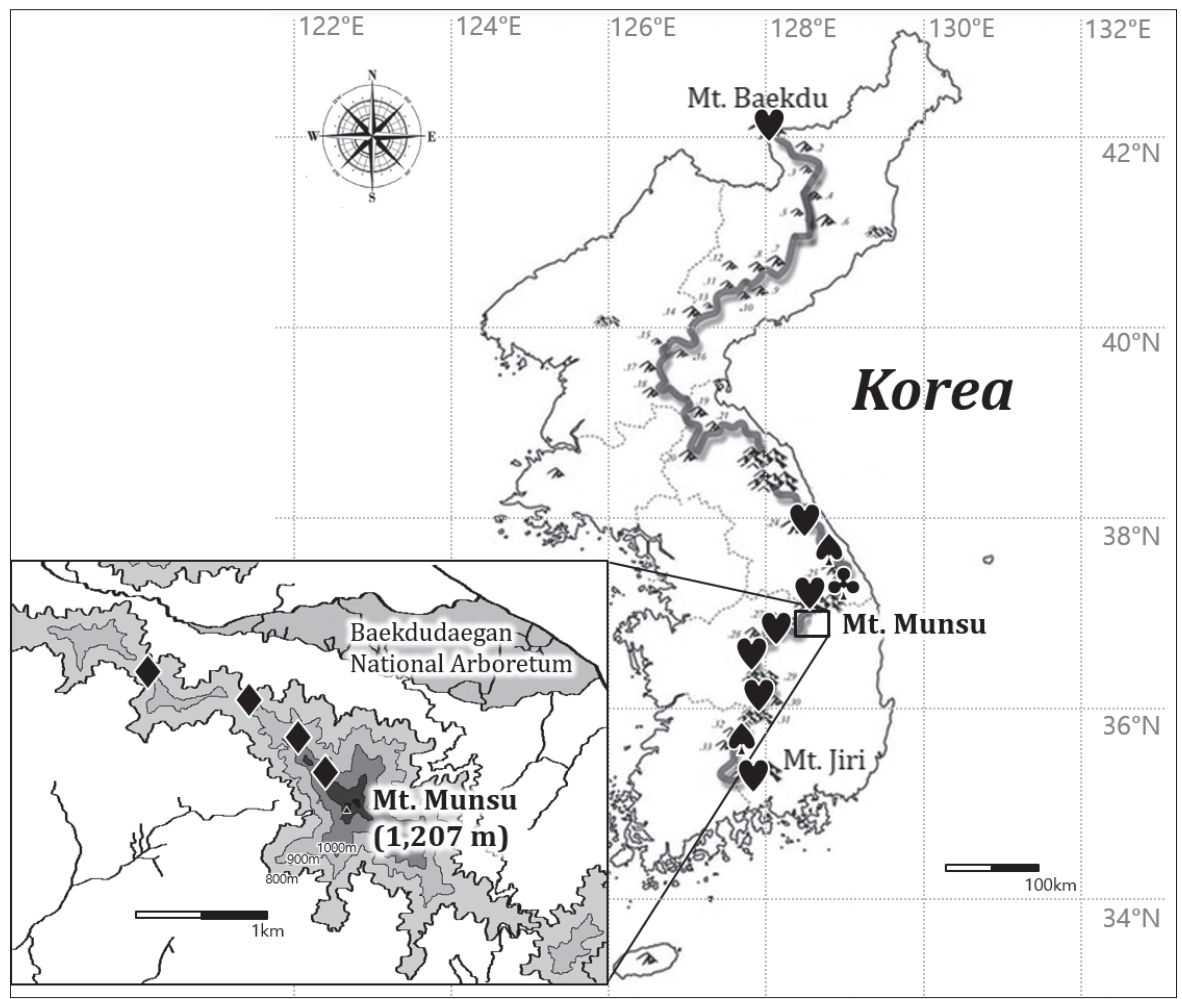

Figure I. Specific collection sites (black symbols) on the Baekdudaegan Mountains (thick gray line on the entire Korea map), for the new species Lecanora baekdudaeganensis $(\downarrow)$, and previously discovered species $L$. hafelliana (⿶), L. loekoesii (\$), and L. pseudosambuci (\$). All Lecanora species reported as new species were detected in the Baekdudaegan Mountains or other mountainous areas just close to the mountains in Korea. 


\section{Materials and methods}

\section{Morphological and chemical analyses}

Hand-cut sections were prepared with a razor blade under a stereomicroscope (Olympus optical SZ51; Olympus, Tokyo, Japan), examined under a compound microscope (Nikon Eclipse E400; Nikon, Tokyo, Japan) and imaged using a software program (AxioVision Release 4.8.2; Carl Zeiss, Jena, Germany) and an Axiocam ERc 5s camera (Carl Zeiss, Jena, Germany) mounted on a Zeiss Axioscope A1 microscope (Carl Zeiss, Jena, Germany). The ascospores were investigated at $1000 \times$ magnification in water. The length and width of the ascospores were measured and the range of spore sizes was shown with average, standard deviation, and number of measured spores. Thin-layer chromatography (TLC) was performed using solvent systems A and C according to standard methods (Orange et al. 2001).

\section{Isolation, DNA extraction, amplification, and sequencing}

Hand-cut sections of ascomata or thallus from all collected specimens were prepared for DNA isolation and DNA was extracted with a NucleoSpin Plant II Kit in line with the manufacturer's instructions (Macherey-Nagel, Düren, Germany). PCR amplification for the internal transcribed spacer region (ITS1-5.8S-ITS2 rDNA) and the mitochondrial small subunit genes was achieved using Bioneer's AccuPower PCR Premix (Bioneer, Daejeon, Korea) in 20- $\mu \mathrm{L}$ tubes and primers ITS5 and ITS4 (White et al. 1990), and mrSSU1 and mrSSU3R (Zoller et al. 1999), respectively. The PCR thermal cycling parameters used were $95^{\circ} \mathrm{C}(15 \mathrm{sec})$, followed by 35 cycles of $95^{\circ} \mathrm{C}(45$ sec), $54^{\circ} \mathrm{C}(45 \mathrm{sec})$, and $72{ }^{\circ} \mathrm{C}(1 \mathrm{~min})$, and a final extension at $72^{\circ} \mathrm{C}(7 \mathrm{~min})$ based on Ekman (2001). DNA sequences were generated by the genomic research company GenoTech (Daejeon, Korea).

\section{Phylogenetic analyses}

All ITS and mtSSU sequences were aligned and edited manually using ClustalW in Bioedit V7.2.6.1 (Hall 1999). All missing and ambiguously aligned data and parsimony-uninformative positions were removed and only parsimony-informative regions were finally analyzed in MEGA X (Stecher et al. 2020). The final alignment comprised 564 (ITS) and 1032 (mtSSU) columns. In them, variable regions were 51 (ITS) and 100 (mtSSU). Finally, the phylogenetically informative regions were 359 (ITS) and 464 (mtSSU). Phylogenetic trees with bootstrap values were obtained in RAxML GUI 2.0 beta (Edler et al. 2019) using the maximum likelihood method with a rapid bootstrap with 1000 bootstrap replications and GTR GAMMA for the substitution matrix. The posterior probabilities were obtained in BEAUti 1.8.0 
and BEAST 1.8.0 (Drummond et al. 2012) using the HKY (Hasegawa, Kishino and Yano) model, as the appropriate model of nucleotide substitution based on the Bayesian Information Criterion (BIC) (Schwarz 1978) as evaluated by bModelTest (Bouchaert and Drummond 2017), empirical base frequencies, gamma for the site heterogeneity model, four categories for gamma, and a 10,000,000 Markov chain Monte Carlo chain length with a 10,000-echo state screening and $1000 \log$ parameters. Then, a consensus tree was constructed in TreeAnnotator 1.8.0 (Drummond and Rambaut 2007) with a burn-in of 5000, no posterior probability limit, a maximum clade credibility tree for the target tree type, and median node heights. All trees were displayed in FigTree 1.4.2 (Rambaut 2014) and edited in Microsoft Paint. The bootstrapping and Bayesian analyses were repeated three times for the result consistency and no significant differences were shown for the tree shapes and branch values. The phylogenetic trees and DNA sequence alignments are deposited in TreeBASE under the study ID 25859.

\section{Results and discussion}

\section{Phylogenetic analyses}

Two independent phylogenetic trees for the Lecanora subfusca group and related species were produced from 122 sequences (61 for ITS, and 61 for mtSSU) from GenBank and with two new sequences (each one for ITS and mtSSU) for the new species (Table 1). The new species was positioned in the L. subfusca group in both ITS and mtSSU trees. In the ITS tree, the new species was located in a clade with L. achroa Nyl., L. allophana (Ach.) Nyl., L. cinereofusca H. Magn., L. horiza (Ach.) Röhl., L. layana Lendemer, L. saxigena Lendemer \& R.C. Harris and L. tropica Zahlbr. (Fig. 2). All species including the new species were in the L. subfusca group except for L. layana which was nevertheless the most closely located to the new species, represented by a bootstrap value of 89 and a posterior probability of 100 for the branch. Many other species, including L. imshaugii in the L. subfusca group, were positioned in different clades and our results did not reveal any close species in the L. subfusca group to the new species. In the mtSSU tree, the new species is located in a clade with L. allophana, L. cenisia Ach., L. expersa Nyl., L. farinaria Borrer, L. horiza, L. imshaugii, L. layana, L. paramerae I. Martínez, Aragón \& Lumbsch, L. pulicaris (Pers.) Ach., L. substerilis Malíček \& Vondrák, L. tropica, and $L$. vainioi Vänskä (Fig. 3). All species including the new species were in the $L$. subfusca group except for L. layana. Except for L. layana, a sorediate species (Lendemer 2015), L. imshangii was the most closely positioned with the new species, represented by a bootstrap value of 90 and a posterior probability of 100 for the branch. Our analysis did not represent any species identical to the new species in the L. subfusca group. 


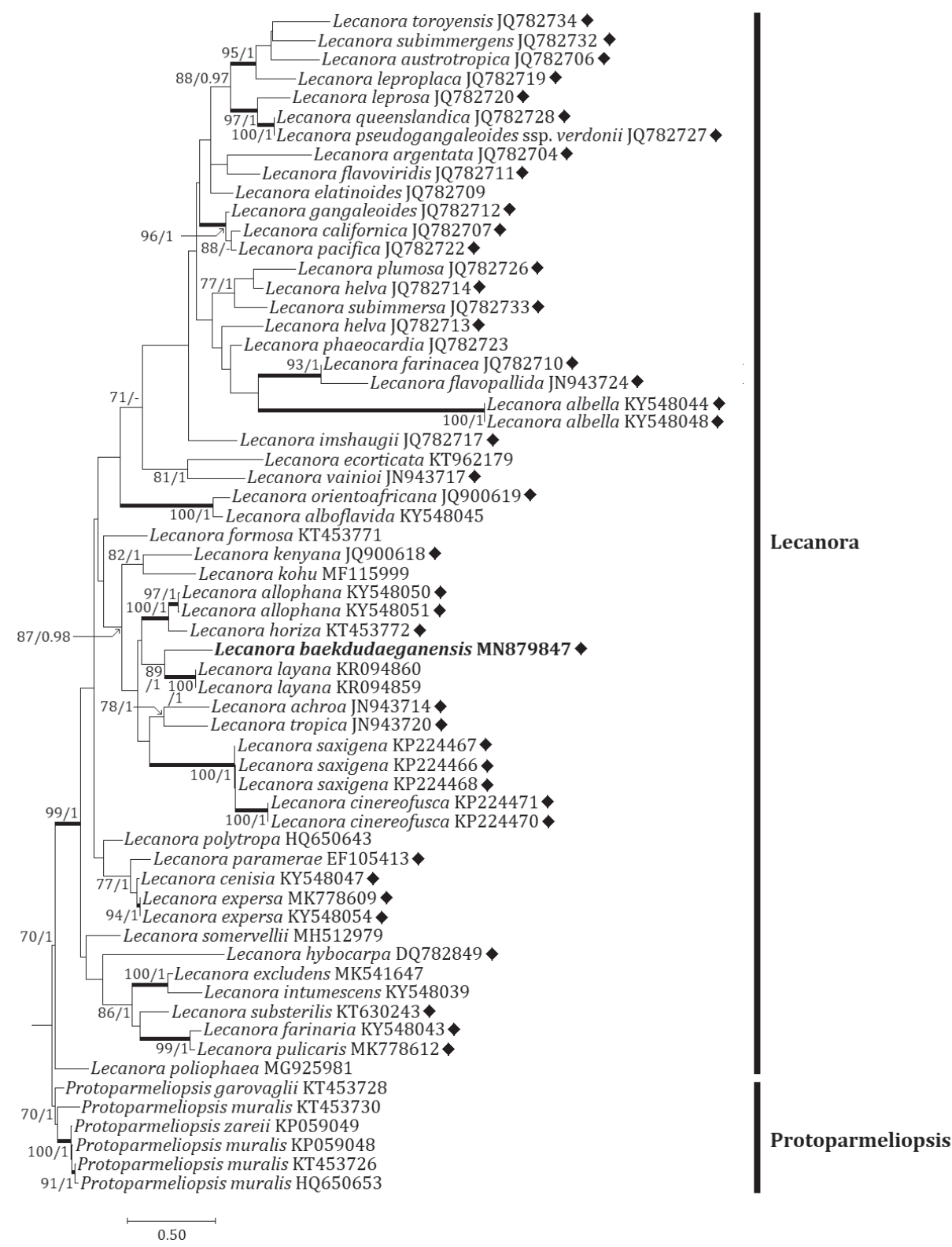

Figure 2. Phylogenetic relationships among comparable species related mainly with the Lecanora subfusca group based on a maximum likelihood analysis of the nuclear ribosomal ITS1-5.8S-ITS2 region. The tree was rooted with several sequences in the genus Protoparmeliopsis. Maximum likelihood bootstrap values $\geq 70 \%$ and posterior probabilities $\geq 95 \%$ are shown above internal branches. Branches with bootstrap values $\geq 90 \%$ are shown in bold. The new species Lecanora baekdudaeganensis is presented in bold, and all species names are followed by GenBank accession numbers. A dash indicates branches with posterior probabilities $<95 \%$. The Lecanora subfusca group is marked with a black diamond ( $\bullet$. Reference Table 1 provides the GenBank accession numbers for the included species and voucher information. 


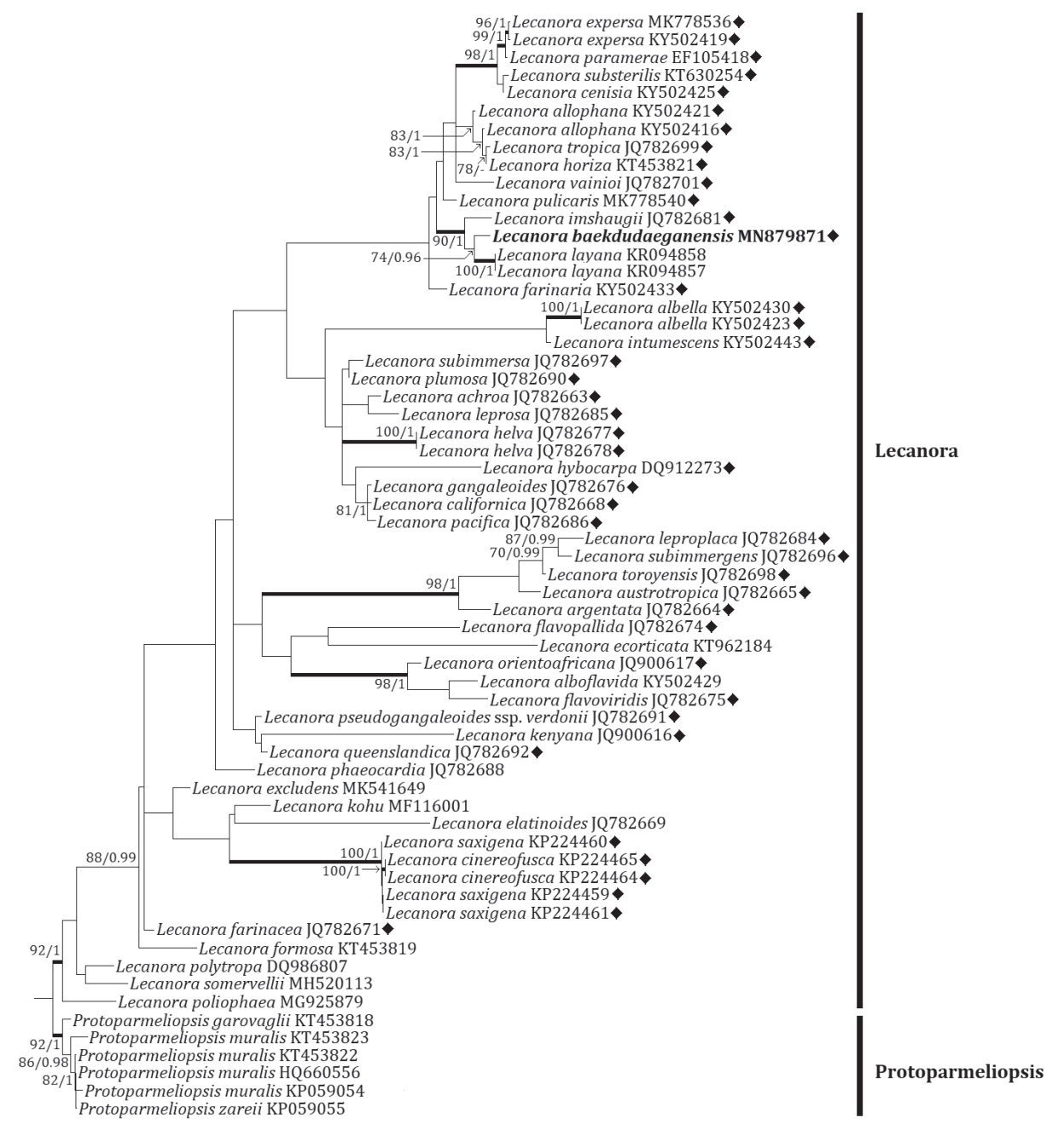

0.50

Figure 3. Phylogenetic relationships among comparable species related mainly with the Lecanora subfusca group based on a maximum likelihood analysis of the mitochondrial small subunit (mtSSU) sequences. The tree was rooted with several sequences in the genus Protoparmeliopsis. Maximum likelihood bootstrap values $\geq 70 \%$ and posterior probabilities $\geq 95 \%$ are shown above internal branches. Branches with bootstrap values $\geq 90 \%$ are shown in bold. The new species Lecanora baekdudaeganensis is presented in bold, and all species names are followed by GenBank accession numbers. A dash indicates branches with posterior probabilities $<95 \%$. The Lecanora subfusca group is marked with a black diamond ( $\downarrow)$. Reference Table 1 provides the GenBank accession numbers for the included species and voucher information 
Table I. Species list and DNA sequence information employed for phylogenetic analysis.

\begin{tabular}{|c|c|c|c|c|}
\hline No. & Species & ID (ITS) & ID (mtSSU) & Voucher \\
\hline 1 & Lecanora achroa & JN943714 & JQ782663 & Papong 6458 \\
\hline 2 & Lecanora albella & KY548044 & KY502430 & Berger 29362 \\
\hline 3 & Lecanora albella & KY548048 & KY502423 & Malicek 7336 \\
\hline 4 & Lecanora alboflavida & KY548045 & KY502429 & Coppins s.n. \\
\hline 5 & Lecanora allophana & KY548050 & KY502421 & Malicek 9626 \\
\hline 6 & Lecanora allophana & KY548051 & KY502416 & Malicek 9491 \\
\hline 7 & Lecanora argentata & JQ782704 & JQ782664 & Papong 6041(F) \\
\hline 8 & Lecanora austrotropica & JQ782706 & JQ782665 & Papong 6407(F) \\
\hline 9 & Lecanora baekdudaeganensis & MN879847 & MN879871 & BDNA-L-0000065 \\
\hline 10 & Lecanora californica & JQ782707 & JQ782668 & Lumbsch $19914 a(F)$ \\
\hline 11 & Lecanora cenisia & KY548047 & KY502425 & Malicek 5869 \\
\hline 12 & Lecanora cinereofusca & KP224470 & KP224465 & Lendemer 34944 (NY) \\
\hline 13 & Lecanora cinereofusca & KP224471 & KP224464 & Lendemer 35007 (NY) \\
\hline 14 & Lecanora ecorticata & KT962179 & KT962184 & NMW $<$ GBR $>$ :C.2015.005.77 \\
\hline 15 & Lecanora elatinoides & JQ782709 & JQ782669 & Lumbsch $19992 d(\mathrm{~F})$ \\
\hline 16 & Lecanora excludens & MK541647 & MK541649 & Palice 21929 \\
\hline 17 & Lecanora expersa & KY548054 & KY502419 & Malicek 9625 \\
\hline 18 & Lecanora expersa & MK778609 & MK778536 & Vondrak 16033 (PRA) \\
\hline 19 & Lecanora farinacea & JQ782710 & JQ782671 & Lumbsch 20022d(F) \\
\hline 20 & Lecanora farinaria & KY548043 & KY502433 & Tonsberg 46170 \\
\hline 21 & Lecanora flavopallida & JN943724 & JQ782674 & Lumbsch 20031a \\
\hline 22 & Lecanora flavoviridis & JQ782711 & JQ782675 & Papong 6539(F) \\
\hline 23 & Lecanora formosa & KT453771 & KT453819 & ZX 20129045-2 \\
\hline 24 & Lecanora gangaleoides & JQ782712 & JQ782676 & Lumbsch $19923 a(F)$ \\
\hline 25 & Lecanora helva & JQ782713 & JQ782677 & Lumbsch $19809 \mathrm{~h}(\mathrm{~F})$ \\
\hline 26 & Lecanora helva & JQ782714 & JQ782678 & Lumbsch $19843 b(\mathrm{~F})$ \\
\hline 27 & Lecanora horiza & KT453772 & KT453821 & Zhao 2015 \\
\hline 28 & Lecanora hybocarpa & DQ782849 & DQ912273 & AFTOL-ID 639 \\
\hline 29 & Lecanora imshaugii & JQ782717 & JQ782681 & Lumbsch $19273 b(\mathrm{~F})$ \\
\hline 30 & Lecanora intumescens & KY548039 & KY502443 & Malicek 8203 \\
\hline 31 & Lecanora kenyana & JQ900618 & JQ900616 & Kirika $1179(\mathrm{~F})$ \\
\hline 32 & Lecanora kohu & MF115999 & MF116001 & UNITEC 7497 \\
\hline 33 & Lecanora layana & KR094859 & KR094857 & Lendemer 37519 (NY) \\
\hline 34 & Lecanora layana & KR094860 & KR094858 & Lendemer 38131 (NY) \\
\hline 35 & Lecanora leproplaca & JQ782719 & JQ782684 & Lumbsch 19815r(F) \\
\hline 36 & Lecanora leprosa & JQ782720 & JQ782685 & Papong 6443(F) \\
\hline 37 & Lecanora orientoafricana & JQ900619 & JQ900617 & Kirika 2205(F) \\
\hline 38 & Lecanora pacifica & JQ782722 & JQ782686 & Lumbsch $19901 \mathrm{c}(\mathrm{F})$ \\
\hline 39 & Lecanora paramerae & EF105413 & EF105418 & Lumbsch s.n. (F) \\
\hline 40 & Lecanora phaeocardia & JQ782723 & JQ782688 & Papong 3473(F) \\
\hline 41 & Lecanora plumosa & JQ782726 & JQ782690 & Papong 6965(F) \\
\hline 42 & Lecanora poliophaea & MG925981 & MG925879 & O:L 200460 \\
\hline 43 & Lecanora polytropa & HQ650643 & DQ986807 & AFTOL-ID 1798 \\
\hline 44 & $\begin{array}{l}\text { Lecanora pseudogangaleoides } \\
\text { subsp. verdonii }\end{array}$ & JQ782727 & JQ782691 & Lumbsch $19103 a(F)$ \\
\hline 45 & Lecanora pulicaris & MK778612 & MK778540 & Malicek 10263 \\
\hline 46 & Lecanora queenslandica & JQ782728 & JQ782692 & Lumbsch 19113j(F) \\
\hline 47 & Lecanora saxigena & KP224466 & KP224459 & Lendemer 32825 (NY) \\
\hline 48 & Lecanora saxigena & KP224467 & KP224460 & Lendemer $25832(\mathrm{NY})$ \\
\hline 49 & Lecanora saxigena & KP224468 & KP224461 & Lendemer 33186 (NY) \\
\hline 50 & Lecanora somervellii & MH512979 & MH520113 & YO 10109 \\
\hline
\end{tabular}




\begin{tabular}{lllcc}
\hline No. & \multicolumn{1}{c}{ Species } & ID (ITS) & ID (mtSSU) & Voucher \\
\hline 51 & Lecanora subimmergens & JQ782732 & JQ782696 & Papong 6431(F) \\
52 & Lecanora subimmersa & JQ782733 & JQ782697 & Lumbsch 19103b(F) \\
53 & Lecanora substerilis & KT630243 & KT630254 & Malicek 202 \\
54 & Lecanora toroyensis & JQ782734 & JQ782698 & Papong 7197(F) \\
55 & Lecanora tropica & JN943720 & JQ782699 & Papong 6440 \\
56 & Lecanora vainioi & JN943717 & JQ782701 & Papong 6957 \\
57 & Protoparmeliopsis garovaglii & KT453728 & KT453818 & Leavitt 089 (BRY-C) \\
58 & Protoparmeliopsis muralis & HQ650653 & HQ660556 & Schmull s. n. \\
59 & Protoparmeliopsis muralis & KP059048 & KP059054 & SK 765 \\
60 & Protoparmeliopsis muralis & KT453726 & KT453822 & Leavitt 143 (BRY-C) \\
61 & Protoparmeliopsis muralis & KT453730 & KT453823 & Vondrak 9413 \\
62 & Protoparmeliopsis zareii & KP059049 & KP059055 & SK 480 \\
& Overall & $\mathbf{6 2}$ & $\mathbf{6 2}$ & \\
\hline
\end{tabular}

DNA sequences for the new species Lecanora baekdudaeganensis (in bold) were generated in this study. All others were obtained from GenBank. The species names are followed by GenBank accession numbers and voucher information. ITS, internal transcribed spacer; mtSSU, mitochondrial small subunit; Voucher, voucher information.

\section{Taxonomy}

\section{Lecanora baekdudaeganensis B.G. Lee \& J-.S. Hur, sp. nov.}

MycoBank No: 833845

Fig. 4

Diagnosis. Lecanora baekdudaeganensis differs from L. imshangii by a darker thallus (bluish, olivish, or pale brownish gray vs. greenish or yellowish gray), brownish disc (vs. reddish brown disc), K-insoluble granules on the surface of epihymenium (vs. absence of granules), shorter hypothecium (15-25 $\mu \mathrm{m}$ vs. 50-75 $\mu \mathrm{m})$, and the presence of oil droplets in the apothecial section.

Type. South Korea, North Gyeongsang Province, Bonghwa-gun, Chunyangmyeon, Mt. Munsu, 36 $59.41^{\prime} \mathrm{N}, 128^{\circ} 48.24^{\prime} \mathrm{E}, 1,005 \mathrm{~m}$ alt., on bark of Quercus mongolica Fisch. ex Ledeb., 29 August 2019, B.G.Lee 2019-000065 (holotype: BDNA-L-0000065!; GenBank MN879847 for ITS and MN879871 for mtSSU); South Korea, North Gyeongsang Province, Bonghwa-gun, Chunyang-myeon, Mt. Munsu, $37^{\circ} 0.31^{\prime} \mathrm{N}, 128^{\circ} 47.39^{\prime} \mathrm{E}$, ca $900 \mathrm{~m}$ alt., on bark of Quercus dentata Thunb., 26 September 2019, B.G.Lee 2019-000135 (paratype: BDNA-L-0000135); SoutH Korea, North Gyeongsang Province, Bonghwa-gun, Chunyang-myeon, Mt. Munsu, $36^{\circ} 59.82^{\prime} \mathrm{N}, 128^{\circ} 46.81^{\prime} \mathrm{E}, 970 \mathrm{~m}$ alt., on bark of Quercus mongolica, 26 August 2019, B.G.Lee 2019-000147 (paratype: BDNA-L-0000147); South KorEA, North Gyeongsang Province, Bonghwa-gun, Chunyang-myeon, Mt. Munsu, 36 59.35'N, $128^{\circ} 46.12^{\prime} \mathrm{E}$, ca 1,075 m alt., on bark of Quercus mongolica, 26 August 2019, B.G.Lee 2019-000151 (paratype: BDNA-L-0000151).

Description. Thallus corticolous, crustose, without lobes, continuous or cracked, rimose to areolate or verruculose, usually rounded or irregular, bluish gray in the beginning (margin) and olivish- or pale brownish-gray when mature (center), not pruinose, $30-70 \mathrm{~mm}$ diam., $100-170 \mu \mathrm{m}$ thick; cortex hyaline to pale yellow or pale brown, 

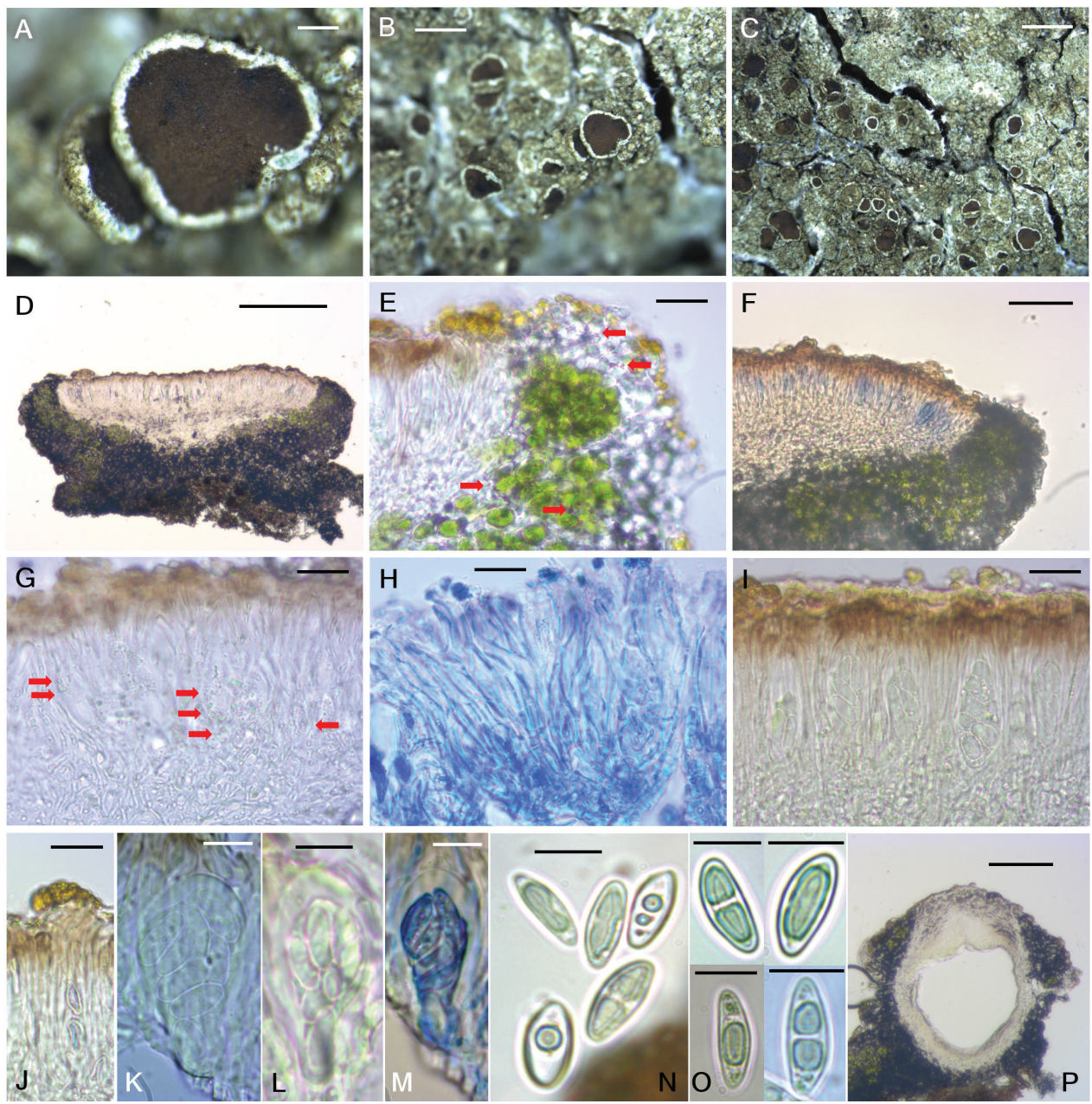

Figure 4. Lecanora baekdudaeganensis (BDNA-L-0000065, holotype) in morphology. A-C Habitus with dark thallus and epruinose apothecia $\mathbf{D}$ sessile apothecia with constricted base in section. Hypothecial base closed by medulla of amphithecium $\mathbf{E}$ well-developed amphithecium with small calcium oxalate crystals (red arrows) not dissolving in $\mathrm{KOH} \mathbf{F}$ apothecial section in Iodine. I- reaction in the beginning then turning slowly to blue or purple-blue hymenium $\mathbf{G}$ oil droplets (red arrows) present in the apothecial section $\mathbf{H}$ anastomosing paraphyses shown in Lactophenol cotton blue $\mathbf{I}$ asci in hymenium $\mathbf{J}$ epihymenium with yellowish granules not dissolving in $\mathrm{KOH} \mathrm{K} \mathbf{~} \mathbf{M}$ 8-spored, clavate asci ( $\mathbf{M}$ in Lactophenol cotton blue) $\mathbf{N}, \mathbf{O}$ ellipsoid ascospores in diverse development stages. Spores biguttulate in the beginning then having a long oil drop by assembly of guttules when mature $\mathbf{P}$ old pycnidia without pycnocodidia. Scale bars: $200 \mu \mathrm{m}(\mathbf{A}, \mathbf{D}), 2 \mathrm{~mm}(\mathbf{B}, \mathbf{C}), 20 \mu \mathrm{m}(\mathbf{E}, \mathbf{G}-\mathbf{J}), 50 \mu \mathrm{m}(\mathbf{F}), 10 \mu \mathrm{m}(\mathbf{K}-\mathbf{O}), 100 \mu \mathrm{m}(\mathbf{P})$.

5-10 $\mu \mathrm{m}$ thick; medulla 20-75 $\mu \mathrm{m}$ thick; photobiont coccoid, forming a distinct algal layer, 45-80 $\mu \mathrm{m}$ thick, cells globose, 8.5-17 × 8-15 $\mu \mathrm{m}$. Prothallus absent.

Apothecia abundant, rounded, smaller and scattered around the margin and larger and aggregated in the center, constricted at the base, $0.2-1.6 \mathrm{~mm}$ diam. Disc flat to 
slightly concave, not pruinose, brown to dark brown from the beginning, 270-430 $\mu \mathrm{m}$ thick; margin persistent, prominent, generally entire or slightly flexuous, some a little crenulate when old, concolorous to thallus. Amphithecium well-developed, with numerous small crystals in both algal-containing and cortical parts (allophana-type) not dissolving in $\mathrm{K}, 60-100 \mu \mathrm{m}$ thick laterally, $110-130 \mu \mathrm{m}$ thick basally; amphithecial cortex distinct, 7-12 $\mu \mathrm{m}$ thick. Parathecium hyaline, indistinct in water, 15-25 $\mu \mathrm{m}$ thick in I. Epihymenium pale yellowish brown to pale brown, with small granules on the surface not dissolving in $\mathrm{K}$, pigment slightly paler in $\mathrm{K}$ but not diluted, without oil droplets, 5-15 $\mu \mathrm{m}$ high. Hymenium hyaline, 50-75 $\mu \mathrm{m}$ high. Subhymenium hyaline, 20-40 $\mu \mathrm{m}$ high. Hypothecium hyaline, coarsely prosoplectenchymatous (periclinal) in the lower and marginal parts and prosoplectenchymatous (irregular) in the upper and central parts, 15-25 $\mu \mathrm{m}$ high. Oil droplets present in hypothecium, subhymenium and the base of hymenium. Hypothecial base not extending or a little extending to the substrate and always closed by medulla of amphithecium. Paraphyses septate, anastomosing, 1-2.5 $\mu \mathrm{m}$ wide, simple or sparsely branched at tips but not, or only

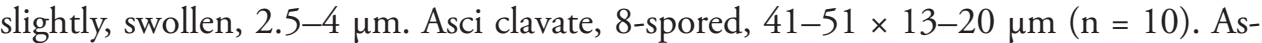
cospores simple, often biguttulate in the beginning then having an oval-shaped oil drop by assembly of guttules when mature, narrowly or widely ellipsoid, or eye-shaped, $10-18.5 \times 4.5-9.5 \mu \mathrm{m}($ mean $=15.2 \times 6.5 ; \mathrm{SD}=1.58(\mathrm{~L}), 1.10(\mathrm{~W}) ; \mathrm{n}=128)$, wall ca $0.5 \mu \mathrm{m}$ thick when exist. Pycnidia only once detected, pale brown at tip, ovoid, $315 \times$ $330 \mu \mathrm{m}$, without conidia as old.

Chemistry. Thallus $\mathrm{K}+$ yellow, $\mathrm{KC}+$ yellow, $\mathrm{C}-$, $\mathrm{Pd}-$. Hymenium I- in the beginning but turning slowly blue or purple-blue, $\mathrm{KI}+$ blue (reaction mainly starting from tholus then the whole ascus), C-, Pd-. UV-. Atranorin, zeorin and an unidentified minor constituent ( $\mathrm{Rf}$ classes $\mathrm{A} 3$ and $\mathrm{C} 3$ in Culberson's standardized thin layer chromatography method (Culberson 1972)), UV- before heating, spot color slightly pale yelloworange after heating, and UV+ pale pink-orange after heating) were detected by TLC.

Distribution and ecology. The species occurs on the bark of Quercus mongolica and $Q$. dentata which are the most dominant tree species on the mountain. This species is currently known from four different sites on the mountain.

Etymology. The species epithet indicates the lichen's geography, namely the main mountains called Baekdudaegan stretching from north to south in the entire Korean Peninsula.

Notes. The new species is classified to the Lecanora subfusca group - allophana type, representing the main characteristics of a crustose thallus without lobes containing atranorin as a major constituent, small calcium oxalate crystals in both algalcontaining and cortical parts of the amphithecium, and trebouxioid photobionts in the thalline margin, dark brown discs, and colorless ellipsoid simple spores in the range of 10-20 × 6-9 $\mu \mathrm{m}$ (Brodo 1984; Miyawaki 1988; Lumbsch 1995; Lumbsch et al. 2003). The new species is compared with Lecanora chionocarpa Hue, L. horiza, L. imshangii, L. japonica Müll. Arg., and L. megalocheila (Hue) H. Miyaw., as those species are in the L. subfusca group with only small crystals in the amphithecium (allophana 
or campestris type) which is defined by the main characteristics such as $\mathrm{K}+$ yellow thallus reaction (containing atranorin), small calcium oxalate crystals in algal-containing and/or cortical parts of amphithecium, and ascospores in the size of 10-20 $\times 6-9 \mu \mathrm{m}$ (Hue 1915; Brodo 1984; Miyawaki 1988; Smith et al. 2009). The new species is most similar to L. imshaugii by a continuous, rimose, verruculose or areolate thallus, the absence of soredia, the absence of a prothallus, apothecia size, and ascospore size (Brodo 1984). However, Lecanora baekdudaeganensis differs from L. imshaugii by a darker thallus (bluish, olivish, or pale brownish gray vs. greenish or yellowish gray), brownish disc (vs. reddish brown disc), K-insoluble granules on the surface of epihymenium (vs. absence of granules), a shorter hypothecium (15-25 $\mu \mathrm{m}$ vs. 50-75 $\mu \mathrm{m})$, and the presence of oil droplets in the apothecial section (Brodo 1984).

The new species is distinguishable from $L$. chionocarpa by a darker thallus (bluish, olivish or pale brownish gray vs. ash gray), the absence of a prothallus (vs. presence of white prothallus), crystals in the amphithecium not dissolving in $\mathrm{K}$ (vs. granular crystals dissolving in $\mathrm{K})$, a shorter hymenium $(50-75 \mu \mathrm{m}$ vs. $75-100 \mu \mathrm{m})$, the presence of oil droplets in hypothecium, subhymenium and the base of hymenium (vs. oil droplets present in epihymenium), shorter asci $(41-51 \times 13-20 \mu \mathrm{m}$ vs. $60-70 \times 13-18 \mu \mathrm{m})$, smaller ascospores $(10-18.5 \times 4.5-9.5 \mu \mathrm{m}$ vs. $15-20 \times 8-11 \mu \mathrm{m})$, thinner ascospore walls $(0.5 \mu \mathrm{m}$ vs. $0.5-1 \mu \mathrm{m})$, and the $\mathrm{Pd}-$ reaction of the thallus and medulla (vs. Pd+ yellowish) (Hue 1915; Miyawaki 1988).

The new species differs from L. horiza by a darker thallus (bluish, olivish or pale brownish gray vs. yellowish white to whitish gray), and the crystals in amphithecium not dissolving in $\mathrm{K}$ (vs. crystals dissolving in $\mathrm{K}$ ) (Smith et al. 2009).

The new species differs from L. japonica by thallus color (bluish, olivish or pale brownish gray vs. dirty greenish to ashy gray), the absence of a prothallus (vs. prothallus with white bundle of hyphae), larger apothecia $(0.2-1.6 \mathrm{~mm}$ vs. up to $1 \mathrm{~mm}$ ), a thicker amphithecium $(60-100 \mu \mathrm{m}$ laterally and $110-130 \mu \mathrm{m}$ basally vs. $5-20 \mu \mathrm{m}$ laterally and $20-50 \mu \mathrm{m}$ basally), the presence of oil droplets in hypothecium, subhymenium and the base of hymenium (vs. oil droplets present in epihymenium), shorter hymenium $(50-75 \mu \mathrm{m}$ vs. $70-80 \mu \mathrm{m})$, a shorter subhymenium $(20-40 \mu \mathrm{m}$ vs. $180-220 \mu \mathrm{m}$ ), a granular epihymenium (vs. non-granular epihymenium), shorter $(41-51 \mu \mathrm{m})$ and constantly 8-spored asci (vs. longer $(50-80 \mu \mathrm{m})$ and 8- or 16-spored asci), the $\mathrm{Pd}-$ reaction of the thallus and medulla (vs. Pd+ pale brown thallus and medulla), and the absence of chloroatranorin (vs. presence of chloroatranorin) (Nylander 1891; Miyawaki 1988; Guderley and Lumbsch 1999).

The new species differs from L. megalocheila by a darker thallus (bluish, olivish or pale brownish gray vs. whitish gray or whitish with green tinge without brownish color), the absence of a prothallus (vs. blackish prothallus), crystals in the amphithecium not dissolving in $\mathrm{K}$ (vs. crystals dissolving in $\mathrm{K}$ ), a shallower hypothecium $(15-25 \mu \mathrm{m}$ vs. $120-150 \mu \mathrm{m})$, wider asci $(41-51 \times 13-20 \mu \mathrm{m}$ vs. $35-50 \times 10-14 \mu \mathrm{m})$, larger ascospores $(10-18.5 \times 4.5-9.5 \mu \mathrm{m}$ vs. $10-14 \times 5-8 \mu \mathrm{m})$, and the Pd- reaction of the thallus and medulla (vs. Pd+ pale yellow thallus and medulla) (Hue 1915; Miyawaki 1988). 


\section{Key to the species in Lecanora of Korea (52 taxa)}

Overall, 56 species have been recorded in the genus Lecanora from Korea (i.e., South Korea (55 spp.) and North Korea (6 spp.) with sharing five species from both countries). However, four of these species are excluded in the key. Lecanora fusanii Hue is regarded as a Caloplaca species because $L$. fusanii (syn. Caloplaca fusanii (Hue) Zahlbr.) has yellow thalli, orange discs, and polarilocular ascospores (Hue 1915). Lecanora subrugosa Nyl. is identical to L. argentata (Ach.) Röhl. based on a molecular analysis (Malíček 2014). Lecanora vulnerata Hue (syn. Caloplaca vulnerata (Hue) Zahlbr.) is supposed to be classified into the family Teloschistaceae because $L$. vulnerata was compared with $L$. heppiana (Müll. Arg.) Hue as a quite similar species, and the former differs from the latter mainly by presence of soredia and $\mathrm{KOH}$ reaction (Hue 1915). The latter is classified in the family Teloschistaceae as a Variospora species at present (Arup et al. 2013). Lecanora muralis (Schreb.) Rabenh., a lobed species, is excluded from the key as it is classified into the genus Protoparmeliopsis. However, one species, L. confusa Almb., is included in the list as the species was discovered in North Korea. A further five species from North Korea, i.e., L. chionocarpa, L. megalocheila, L. polytropa (Ehrh.) Rabenh., L. rubina (Hoffm.) Ach., and L. subrubra Hue (syn. L. japonica), were previously discovered in South Korea as well.

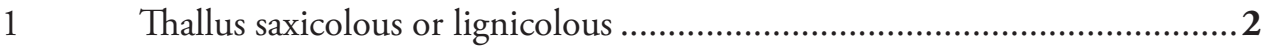

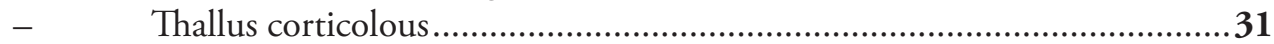

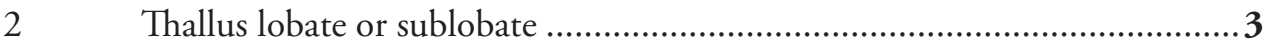

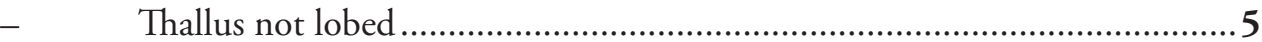

3 Disc dark, ruby-colored .......................................................... L. rubina

- $\quad$ Disc light-colored, pale pink, greenish brown to yellow-brown ................... 4

4 Thallus usually areolate or sometimes sublobate, paraphyses tips swollen up to $3 \mu \mathrm{m}$ wide, conidia $20-25 \times 1 \mu \mathrm{m}$, thallus $\mathrm{Pd}+$ orange.

L. albescens (Myriolecis albescens)

Thallus lobate, resetting, paraphyses tips hardly swollen, conidia absent, thallus Pd-

L. valesiaca

Thallus inconspicuous, immersed or with dispersed areoles, ascospores 10-14 $\times 5-6.5 \mu \mathrm{m}$, thallus $\mathrm{UV}-$.

L. polytropa

Thallus clearly visible

On calcareous rocks or wood/logs.

On non-calcareous rocks

Common on wood/logs, thalline margin excluded finally

Only on calcareous rocks, thalline margin persistent Thallus starkly white or pale gray, apothecia $0.1-0.7 \mathrm{~mm}$ diam., thallus $\mathrm{Pd}+$ orange.

L. albescens (Myriolecis albescens)

Thallus gray to blackened, apothecia $0.5-1.4 \mathrm{~mm}$ diam., thallus $\mathrm{Pd}-$ 
10 Prothallus green-black, thalline margin \pm excluded, disc densely gray pruinose, epihymenium green- or blue-gray, containing zeorin, 士gangaleoidin, and usnic acid.

L. sulphurea

- $\quad$ Prothallus whitish, thalline margin persistent, containing small or large crystals, disc slightly or faintly pruinose, epihymenium brownish, containing 土chloratranorin

11

11 Thalline margin with small, irregular crystals $(<10 \mu \mathrm{m}$ diam.) not dissolving in $\mathrm{K}$, thallus white to yellow-white, $\mathrm{K}+$ indistinct yellow

L. horiza Thalline margin with large crystals (>10 $\mu \mathrm{m}$ diam.), thallus grayish, yellowish or brownish, $\mathrm{K}+$ yellow or yellow turning to red..... 12

12 Thallus not glossy, apothecia 1-2 mm diam., disc yellow-brown, red-brown to black, ascospores 9-15 × 6-8.5 $\mu \mathrm{m}$, containing \pm roccellic acid and norstictic acid

L. cenisia

Thallus somewhat glossy, apothecia $0.4-0.7 \mathrm{~mm}$ diam., disc waxy or pale to greenish orange, ascospores 8-11.5 × 4-6.5 $\mu \mathrm{m}$

L. plumosa

Disc blackish, epihymenium bluish or greenish, not reddish, orangish or brownish

Disc yellowish to brownish, epihymenium yellowish to brownish 16 Thallus grayish white to white, prothallus blackish when present, ascospores smaller, 7-13 × 4-6.5 $\mu \mathrm{m}$, thallus $\mathrm{Pd}+$ yellow, medulla $\mathrm{Pd}+$ pale yellow

L. oreinoides

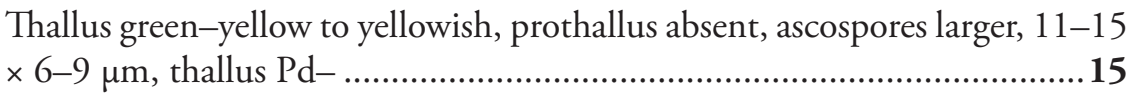
Thallus not shining, apothecia $0.7-4 \mathrm{~mm}$ diam., disc not shining, hymenium 80-110 $\mu \mathrm{m}$ high, ascospores $11-15 \times 6-9 \mu \mathrm{m}$

L. decorata Thallus somewhat shining and waxy, apothecia ca $0.5 \mathrm{~mm}$ diam., disc shining, hymenium 55-65 $\mu \mathrm{m}$ high, ascospores $11-13 \times 6-7 \mu \mathrm{m} .$. L. marginata Disc pale to green-brown or black-green

Disc pale brown, red-brown to dark brown

17 Thallus somewhat glossy, prothallus whitish to whitish gray when present, apothecia $0.4-0.7 \mathrm{~mm}$ diam., disc waxy, pale to greenish orange, ascospores 8-11.5 $\times 4-6.5 \mu \mathrm{m}$, thallus $\mathrm{Pd}+$ pale orange, containing atranorin and chloratranorin

L. plumosa

- $\quad$ Thallus not glossy, prothallus black when present, apothecia 0.3-1 mm diam., disc not waxy, pale yellow to greenish brown or greenish black, ascospores 10-14 × 5-7 $\mu \mathrm{m}$, thallus $\mathrm{Pd}-$, containing usnic acid and zeorin 18

18 Thallus continuous and well-developed, disc green-brown to green-black, epihymenium green-brown to brown, hymenium 60-70 $\mu \mathrm{m}$ high, pycnoconidia $23-25 \times 0.5-1 \mu \mathrm{m}$, thallus $\mathrm{UV}+$ dull orange.

L. intricata Thallus inconspicuous with dispersed areoles, disc pale yellow to pale brown, epihymenium hyaline to yellow- or red-brown, hymenium 45-60 $\mu \mathrm{m}$ high, pycnoconidia $18-22 \times 1 \mu \mathrm{m}$, thallus $\mathrm{UV}_{-}$, containing rangiformic acid and 土eulecanoral 
19 Thallus richly sorediate, disc dark brown and shiny. L. ussuriensis Thallus not sorediate, disc not shiny

20 Thallus pale to medium yellow or yellow-green, not white or gray

L. frustulosa

- Thallus pale to white, gray or dark gray.....

21 Apothecia smaller, up to ca $0.5 \mathrm{~mm}$ diam. 22 Apothecia larger, $0.5-2.5 \mathrm{~mm}$ diam.

22 Thallus medium to dark gray, epihymenium yellow to brown, ascospores 9-15 × 4-6 $\mu \mathrm{m}$, often guttulate and appearing 1-septate, reaction all negative else epihymenium $\mathrm{K}+$ yellow

L. helicopis

Thallus grayish white, epihymenium brownish red, ascospores $12-13 \times 5-6$ $\mu \mathrm{m}$, thallus $\mathrm{K}+$ pale yellow, epihymenium $\mathrm{K}-$

Prothallus absent

Thallus pale, gray to dark gray, thalline margin with irregular or large crystals

Thallus white, grayish white or yellowish white, thalline margin with small crystals

Ascospores narrower, $10-15 \times 5-7 \mu \mathrm{m}$, thallus $\mathrm{Pd}-\ldots \ldots \ldots . .$. L. subimmergens Ascospores wider, 9-15 $\times 6-8.5 \mu \mathrm{m}$, thallus $\mathrm{Pd}+$ weakly yellow or yellow turning to red 26

Epihymenium pale orange to red-brown without granules, paraphyses tips red-brown, asci 50-60 × 12-21 $\mu \mathrm{m}$, containing zeorin

L. campestris Epihymenium brown to olivaceous brown with coarse granules dissolving in $\mathrm{K}$, paraphyses tips olivaceous, asci 45-50 × 7-9 $\mu \mathrm{m}$, containing \pm roccellic acid and \pm norstictic acid

L. cenisia

27 Disc orange, red-orange to red-brown, thalline margin with small irregular crystals not dissolving in $\mathrm{K}$, hypothecium without oil droplets, thallus Pd-, containing \pm chloratranorin

L. horiza

- $\quad$ Disc brown to dark brown, thalline margin with small and large crystals dissolving in $\mathrm{K}$, hypothecium inspersed with oil droplets, thallus $\mathrm{Pd}+$ light orange, containing zeorin

L. melacarpella

Ascospores smaller, $8-12 \times 4.5-5 \mu \mathrm{m}$

L. orientalis

Ascospores larger, 9-15 × 5-8.5 $\mu \mathrm{m}$.....

29 Thalline margin with only large crystals not dissolving in $\mathrm{K}$, thallus yellowish gray to whitish gray, ascospores narrower, $9-15 \times 5-7 \mu \mathrm{m}$, prothallus absent...

L. pseudistera

Thalline margin with small crystals, thallus whitish to grayish white, ascospores wider, $10.5-15 \times 6.5-8.5 \mu \mathrm{m}$, prothallus white when present.....30 
30 Disc orange, red-orange or red-brown, thalline margin with small irregular crystals not dissolving in $\mathrm{K}$, ascospores $12-15 \times 6.5-8.5 \mu \mathrm{m}$, thallus $\mathrm{K}+$ indistinct yellow, $\mathrm{Pd}-$, containing \pm chloratranorin

L. horiza

- $\quad$ Disc brown to dark brown, thalline margin with small and large crystals dissolving in $\mathrm{K}$, ascospores $10.5-13.5 \times 7.5-8.5 \mu \mathrm{m}$, thallus $\mathrm{K}+$ yellow, Pd+ light orange, containing zeorin........................................ L. melacarpella

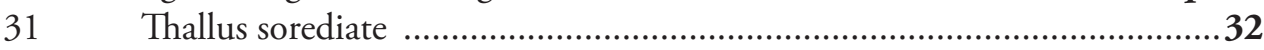

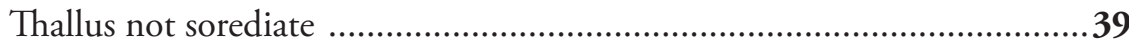

32 Apothecia absent or rarely seen ...................................................33

- Apothecia present

.35

33 Thallus UV-, apothecia not seen, containing stictic acid

L. layana

Thallus UV+ pale orange or ice blue, apothecia rarely seen.

34

34 Thallus pale gray, prothallus pale gray, apothecia not pruinose, ascospores $7-10 \times 3-4 \mu \mathrm{m}$, thallus $\mathrm{Pd} \pm$ yellow, UV + pale orange, containing chloratranorin

L. barkmaniana

Thallus yellow to greenish, occasionally with blue or gray tints, prothallus white and fibrous, often with one or two blue-gray zones, apothecia faintly or heavily white pruinose, ascospores 11-14 × 6-9 $\mu \mathrm{m}$, thallus Pd-, UV+ ice blue (or violet), containing usnic acid and porphyrilic acid

L. thysanophora

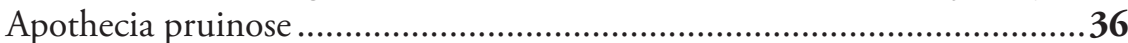

Apothecia not pruinose. 38

Ascospores smaller, 11-14 × 6-9 $\mu \mathrm{m}$

L. thysanophora

Ascospores larger, $15-24 \times 7-12 \mu \mathrm{m}$

37 Thallus white or yellowish white, asci 8- or 6-spored, ascospores $15-22 \times$ $7-12 \mu \mathrm{m}$

L. pachycheila

Thallus yellowish-blue green, asci 8-spored, ascospores 15-20 ×9-12 $\mu \mathrm{m}$...

L. sibirica

Thallus pale gray, apothecia $0.4-0.7 \mathrm{~mm}$ diam., ascospores 7-10 × 3-4 $\mu \mathrm{m}$.

L. barkmaniana

Thallus yellowish gray to greenish gray, apothecia $0.2-0.6 \mathrm{~mm}$ diam., ascospores $12-15.5 \times 6-8.5 \mu \mathrm{m}$, containing chodatin, demethlchodatin, and thiophanic acid

L. leproplaca

39 Prothallus distinct, whitish, grayish or blackish ..............................40

Prothallus indistinct or absent..................................................4 47

$40 \quad$ Prothallus whitish or grayish, but not blackish...................................41

Prothallus blackish ..................................................................44 44

41 Thalline margin with large crystals (> $10 \mu \mathrm{m}$ diam.) not dissolving in $\mathrm{K} \ldots \mathbf{4 2}$

Thalline margin with small crystals $(<10 \mu \mathrm{m}$ diam.).

42 Apothecia pale orange to yellowish brown, sometimes slightly pruinose on disc, epihymenium hyaline to yellow-brown with numerous small crystals 
(chlarotera-type), ascospores 9-13 × 5-7 $\mu \mathrm{m}$, containing gangaleoidin, chloratranorin, chlorolecideoidin, leoidin, and norgangaleoidin L. leprosa Apothecia dark reddish brown to brownish black, not pruinose, epihymenium reddish brown with fine brown granules (pulicaris-type), ascospores 11-14 × 6-8 $\mu \mathrm{m}$, containing fumarprotocetraric acid, \pm roccellic acid

L. pulicaris

Subhymenium 60-80 $\mu \mathrm{m}$ high, asci consistently 8-spored, ascospores $15-20$ $\times 8-11 \mu \mathrm{m}$, pycnidia brown-black with pycnoconidia $20-25 \times 0.5 \mu \mathrm{m}$, containing zeorin

L. chionocarpa Subhymenium 180-220 $\mu \mathrm{m}$ high, asci 8-spored or 16-spored, ascospores $12-16 \times 6-8 \mu \mathrm{m}$, pycnidia absent

L. japonica Thalline margin with granular crystals dissolving in K .........L. megalocheila Thalline margin with large crystals not dissolving in $\mathrm{K}$

45 Disc paler, orange-brown or pale red-brown, flat to slightly convex, epihymenium inspersed with coarse granules, thallus $\mathrm{Pd}-$, containing pannarin, \pm placodialic acid, and \pm roccellic acid

L. cinereofusca epihymenium without granules, thallus Pd+ faintly yellow Paraphyses tips reddish brown (or faintly yellow), asci wider, 45-55 × 18-22 $\mu \mathrm{m}$, ascospores larger, 11.5-14.5 $\times 6-8.5 \mu \mathrm{m}$, containing gangaleoidin and usually traces of californin

L. argentata Paraphyses tips dark brown, asci narrower, 50-60 × 8-12 $\mu \mathrm{m}$, ascospores smaller, 9-14 × 5-8 $\mu \mathrm{m}$, containing zeorin. L. iseana Thalline margin finally excluded .48 Thalline margin permanent 51

48 Disc pruinose, thallus not corticate, containing decarboxysquamatic acid.....

L. strobilina

Disc not pruinose, thallus corticate, decarboxysquamatic acid absent. 49 Asci 16- or 32-spored, thallus $\mathrm{C}-$, $\mathrm{K}-$-, $\mathrm{KC}-$, containing no substance

L. sambuci

Asci 8-spored, thallus $\mathrm{C} \pm$ orange, $\mathrm{KC} \pm$ yellow to orange, containing usnic acid, zeorin and xanthones.

Thallus yellow-green to gray-green, disc pale yellow to greenish, when young the exciple crenulate and containing algae. Thallus yellowish-white to greenish black, disc pinkish brown to greenish black, when young the exciple smooth and lacking algae Disc and epihymenium darker, brown, red-brown to dark brown, not pruinose

Disc or epihymenium paler, pinkish, pale orangish, green-brown, yellowbrown, orange-brown to pale red-brown, pruinose or not. Thalline margin with small crystals (allophana-type) not dissolving in K...53 Thalline margin with large crystals (pulicaris-type) 
53 Thallus darker, bluish, olivish or pale brownish gray, disc brownish, amphithecial cortex present, epihymenium with granules on the surface not dissolving in $\mathrm{K}$, hypothecium shorter, 15-25 $\mu \mathrm{m}$ high, oil droplets present in apothecial section.

L. baekdudaeganensis

- $\quad$ Thallus paler, greenish or yellowish gray, disc reddish brown, amphithecial cortex indistinct or absent, epihymenium without granules, hypothecium taller, 50-75 $\mu \mathrm{m}$ high, oil droplets absent

Epihymenium with coarse, hyaline to brown granules (chlarotera-type), ascospores $8-12 \times 4.5-7 \mu \mathrm{m}$ Apothecia smaller, $0.4-0.8 \mathrm{~mm}$ diam., ascospores $11.5-14.5 \times 6-8.5 \mu \mathrm{m}$, thallus $\mathrm{Pd}+$ weakly yellow, containing gangaleoidin

L. argentata Apothecia larger, up to $1.6 \mathrm{~mm}$ diam., ascospores $10.5-16.5 \times 6-9.5 \mu \mathrm{m}$, thallus $\mathrm{Pd}-$, containing zeorin

L. perplexa Apothecia not constricted at base, disc slightly convex when mature, hymenium including subhymenium 80-100 $\mu \mathrm{m}$ high, hypothecium 120-150 $\mu \mathrm{m}$ high, asci 40-50 × 7-12 $\mu \mathrm{m}$, ascospores 8-10 × 4.5-6 $\mu \mathrm{m}$, pycnidia absent, thallus $\mathrm{Pd}+$ orange

L. nipponica

Apothecia slightly constricted at base, disc extremely convex when mature, hymenium including subhymenium 150-200 $\mu \mathrm{m}$ high, hypothecium 70$110 \mu \mathrm{m}$ high, asci 50-60 × 10-15 $\mu \mathrm{m}$, ascospores 9-12 $\times 6-7 \mu \mathrm{m}$, pycnidia present, thallus $\mathrm{Pd}-$

L. sulcata

Ascospores smaller, 7-10 × 4-5 $\mu \mathrm{m}$, thallus $\mathrm{C}-, \mathrm{K}-, \mathrm{KC}-$ (or $\mathrm{KC}+$ yellow), $\mathrm{Pd}-$.

Ascospores larger, 9-15 × 5-9.5 $\mu \mathrm{m}$, thallus $\mathrm{K}+$ yellow, $\mathrm{Pd}+$ yellow-orange to orange.

59 Ascospores 9-11.5 × 5-7 $\mu \mathrm{m}$, thallus $\mathrm{K}+$ weakly yellow, $\mathrm{Pd}+$ sulphur yellow, thallus areoles somewhat shiny, containing psoromic acid and usnic acid......

Ascospores 9-15 × 5-9.5 $\mu \mathrm{m}$, thallus $\mathrm{K}+$ distinct yellow or yellow turning to red, $\mathrm{Pd}+$ pale yellow to yellow orange, thallus not shiny. Thalline margin with numerous small crystals (allophana-type), containing \pm stictic acid

61 Disc carneous to pinkish, flat to convex, heavily pruinose, epihymenium gray-brown, thallus $\mathrm{K}+$ yellow or yellow turning to red, $\mathrm{Pd}+$ orange, containing chloratranorin, \pm norstictic acid, \pm protocetraric acid, \pm virensic acid, \pm connorstictic acid, \pm conprotocetraric acid, and \pm salazinic acid

L. caesiorubella

- $\quad$ Disc yellowish brown to dark reddish brown, flat to slightly concave, slightly pruinose, epihymenium dark brown to blackish brown, thallus $\mathrm{K}+$ yellow, 
$\mathrm{Pd}+$ pale yellow, containing hafellic acid, zeorin, and usnic acid

62

Thallus pale to medium gray, apothecia larger, $0.7-1.5 \mathrm{~mm}$ diam., disc not pruinose, epihymenium red-brown, paraphyses anastomosed, ascospores larger, 10-14.5 × 7-8.5 $\mu \mathrm{m}$, containing pannarin and \pm placodialic acid

L. cinereofusca

- Thallus yellow-white to yellow gray, apothecia smaller, 0.2-1 mm diam., disc slightly pruinose, epihymenium pale to red-brown, paraphyses sparsely branched, ascospores smaller, 9-13.5 × 5-7 $\mu \mathrm{m}$

63 Apothecia $0.5-1 \mathrm{~mm}$ diam., prothallus absent, thallus Pd-, containing \pm roccellic acid and \pm fatty acid

L. bybocarpa

- $\quad$ Apothecia $0.2-0.7 \mathrm{~mm}$ diam., prothallus gray when present, thallus $\mathrm{Pd}+$ pale orange, containing gangaleoidin, chloratranorin, chlorolecideoidin, leoidin, and norgangaleoidin

L. leprosa

\section{Acknowledgements}

This work was supported by a grant from the Korean National Research Resource Center Program (NRF-2017M3A9B8069471) and the Korean Forest Service Program through the Korea National Arboretum (KNA1-1-22, 17-2).

\section{References}

Arup U, Søchting U, Frödén P (2013) A new taxonomy of the family Teloschistaceae. Nordic Journal of Botany 31(1): 016-083. https://doi.org/10.1111/j.1756-1051.2013.00062.x

Bouckaert RR, Drummond AJ (2017) bModelTest: Bayesian phylogenetic site model averaging and model comparison. BMC evolutionary biology 17(1): 42. https://doi.org/10.1186/ s12862-017-0890-6

Brodo IM (1984) The North American species of the Lecanora subfusca group. Beihefte zur Nova Hedwigia 79: 63-185.

Culberson CF (1972) Improved conditions and new data for identification of lichen products by standardized thin-layer chromatographic method. Journal of Chromatography A 72(1): 113-125. https://doi.org/10.1016/0021-9673(72)80013-X

Drummond AJ, Rambaut A (2007) BEAST: Bayesian evolutionary analysis by sampling trees. BMC evolutionary biology 7(1): 214. https://doi.org/10.1186/1471-2148-7-214

Drummond AJ, Suchard MA, Xie D, Rambaut A (2012) Bayesian phylogenetics with BEAUti and the BEAST 1.7. Molecular biology and evolution 29(8): 1969-1973. https://doi. org/10.1093/molbev/mss075

Edler D, Klein J, Antonelli A, Silvestro D (2019) raxmlGUI 2.0 beta: a graphical interface and toolkit for phylogenetic analyses using RAxML. bioRxiv. https://doi.org/10.1101/800912 
Ekman (2001) Molecular phylogeny of the Bacidiaceae (Lecanorales, lichenized Ascomycota). Mycological Research 105: 783-97. https://doi.org/10.1017/S0953756201004269

Guderley R, Lumbsch HT (1999) Notes on multispored species of Lecanora sensu stricto. The Lichenologist 31(2): 197-203. https://doi.org/10.1006/lich.1998.0190

Hall TA (1999) BioEdit: A User-Friendly Biological Sequence Alignment Editor and Analysis Program for Windows 95/98/NT. Nucleic Acids Symposium Series 41: 95-98.

Hue AM (1915) Lichens novos vel melius cognitos. Annales Mycologici 13: 73-103.

Kondratyuk SY, Lőkös L, Halda JP, Haji Moniri M, Farkas E, Park JS, Lee BG, Oh SO, Hur JS (2016) New and noteworthy lichen-forming and lichenicolous fungi 4. Acta Botanica Hungarica 58(1-2): 75-136. https://doi.org/10.1556/034.58.2016.1-2.4

Korea Forest Service (2019) The Ecological Map of the Baekdudaegan Mountains [Brochure]. Daejeon, South Korea: Korea Forest Service.

Lendemer JC (2015) Lecanora layana (Lecanoraceae), a new sorediate species widespread in temperate eastern North America. The Bryologist 145-153. https://doi.org/10.1639/00072745-118.2.145

Lumbsch HT (1995) A new species in the Lecanora subfusca group containing usnic acid in addition to atranorin. The Lichenologist 27(3): 161-167. https://doi.org/10.1016/S00242829(95) 80015-8

Lumbsch HT, Messuti MI, Nash TH (2003) New or Overlooked Species in the Lecanora subfusca Group from Southwestern North America (Lecanorales, Ascomycotina). The Bryologist 106(4): 552-559. https://doi.org/10.1639/0007-2745(2003)106[552:NOOSIT]2.0. $\mathrm{CO} ; 2$

Lü L, Joshi Y, Lumbsch HT, Wang HY, Koh YJ, Hur JS (2011) New and noteworthy species of the lichen genus Lecanora (Ascomycota; Lecanoraceae) from South Korea. The Lichenologist 43(4): 321-329. https://doi.org/10.1017/S0024282911000144

Malíček J (2014) A revision of the epiphytic species of the Lecanora subfusca group (Lecanoraceae, Ascomycota) in the Czech Republic. The Lichenologist 46(4): 489-513. https:// doi.org/10.1017/S0024282914000139

Miyawaki H (1988) Studies on the Lecanora subfusca group in Japan. Journal of the Hattori Botanical Laboratory 64: 271-326.

Nylander W (1891) Sertum lichenaeæ tropicæ e Labaun et Singapore. e typis P. Schmidt.

Orange A, James PW, White FJ (2001) Microchemical Methods for the Identification of Lichens. The British Lichen Society, London, UK.

Rambaut A (2014) FigTree v1.4.2. Edinburgh: University of Edinburgh. http://tree.bio.ed.ac. uk/software/figtree

Schwarz G (1978) Estimating the dimension of a model. Annals of Statistics 6: 461-464. https://doi.org/10.1214/aos/1176344136

Smith CW, Aptroot A, Coppins BJ, Fletcher A, Gilbert OL, James PW, Wolseley PA (2009) The lichens of Great Britain and Ireland. The British Lichen Society, London, UK.

Stecher G, Tamura K, Kumar S (2020) Molecular Evolutionary Genetics Analysis (MEGA) for macOS, Molecular Biology and Evolution 37(4): 1237-1239. https://oi.org/10.1093/ molbev/msz312 
White TJ, Bruns T, Lee S, Taylor JW (1990) Amplification and direct sequencing of fungal ribosomal RNA genes for phylogenetics. PCR protocols: a guide to methods and applications 18(1): 315-322. https://doi.org/10.1016/B978-0-12-372180-8.50042-1

Zoller S, Scheidegger C, Sperisen C (1999) PCR primers for the amplification of mitochondrial small subunit ribosomal DNA of lichen-forming ascomycetes. The Lichenologist 31(5): 511-516. https://doi.org/10.1006/lich.1999.0220 